\title{
Achieving Change Requirements of Feature Models by an Evolutionary Approach
}

\author{
Paolo Arcaini \\ National Institute of Informatics \\ Tokyo, Japan \\ arcaini@nii.ac.jp
}

Extended Abstract

\author{
Angelo Gargantini \\ University of Bergamo \\ Bergamo, Italy \\ angelo.gargantini@unibg.it
}

\author{
Marco Radavelli \\ University of Bergamo \\ Bergamo, Italy \\ marco.radavelli@unibg.it
}

\section{CCS CONCEPTS}

- Software and its engineering $\rightarrow$ Software product lines; Search-based software engineering; • Theory of computation $\rightarrow$ Evolutionary algorithms;

\section{KEYWORDS}

feature models, software product lines, mutation, search-based software engineering

ACM Reference Format:

Paolo Arcaini, Angelo Gargantini, and Marco Radavelli. 2019. Achieving Change Requirements of Feature Models by an Evolutionary Approach: Extended Abstract. In 23rd International Systems and Software Product Line Conference - Volume A (SPLC '19), September 9-13, 2019, Paris, France. ACM, New York, NY, USA, 1 page. https://doi.org/10.1145/3336294.3342375

\section{DESCRIPTION OF THE JOURNAL-FIRST PAPER}

Software Product Lines (SPLs) are families of products that share some common features, and differ on some others. The variability of SPLs is usually described at design time by using variability models; one of the main used variability models are feature models (FMs).

Overtime, feature models need to be updated in order to avoid the risk of having a model with wrong features and/or wrong constraints. Two main causes for change requirements exist: either the model is incorrect (it excludes/includes some products that should be included/excluded), or the SPL has changed. The change requirements come from different sources: failing tests identifying configurations evaluated not correctly, or business requirements to add new products, to allow new features, to not support some products any more, and so on. All these change requirements identify configurations/features to add or remove, but do not identify a way to modify the feature model to achieve them. Manually updating a feature model to achieve all the change requirements can be particularly difficult and, in any case, error-prone and time consuming.

In the corresponding journal-first paper [1], we propose an approach (shown in Fig. 1) to automatically update a feature model upon change requirements. The user must only specify an update request, based on the change requirements coming from testing or from business requirements. The update request is composed of

Permission to make digital or hard copies of part or all of this work for personal or classroom use is granted without fee provided that copies are not made or distributed for profit or commercial advantage and that copies bear this notice and the full citation on the first page. Copyrights for third-party components of this work must be honored.

For all other uses, contact the owner/author(s).

SPLC '19, September 9-13, 2019, Paris, France

(C) 2019 Copyright held by the owner/author(s)

ACM ISBN 978-1-4503-7138-4/19/09.

https://doi.org/10.1145/3336294.3342375

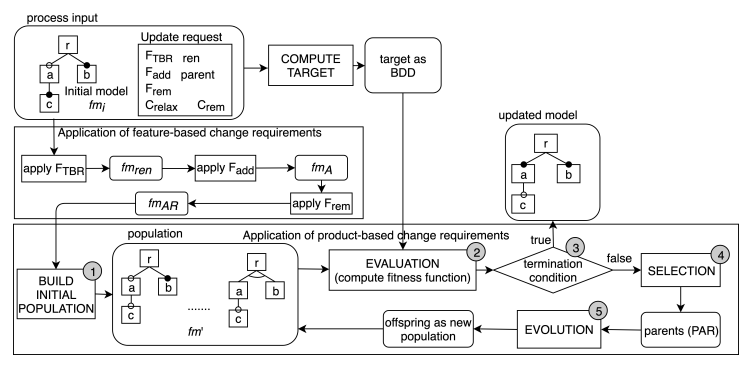

Figure 1: Proposed evolutionary approach

three kinds of feature-based change requirements and two kinds of product-based ones; the feature-based ones consist in features that must be renamed, and features that must be added to and removed from the products of the original feature model; the product-based ones, instead, consist in configurations that should be no more accepted as products by the final model, and configurations that should instead be accepted as new products. Starting from the update request, the approach tries to apply the feature-based change requirements directly on the starting model; however, some of these requirements could be not completely fulfilled. Then, by means of an evolutionary algorithm, the approach tries to obtain a feature model that captures all the change requirements: the feature-based ones not fulfilled in the previous phase, and the product-based ones. The process iteratively generates, from the current population of candidate solutions, a new population of feature models by mutation. Population members are evaluated considering primarily the percentage of correctly evaluated configurations, and secondly a measure of the structural complexity of the model, defined in terms of number of cross-tree constraints. When a correct model is found or some other termination condition holds, the process terminates returning as final model the one having the highest fitness value.

The approach has been evaluated on real-world feature models; experiments show that, on average, around $89 \%$ of requested changes are applied.

\section{ACKNOWLEDGMENTS}

P. Arcaini is supported by ERATO HASUO Metamathematics for Systems Design Project (No. JPMJER1603), JST. Funding Reference number: $10.13039 / 501100009024$ ERATO.

\section{REFERENCES}

[1] Paolo Arcaini, Angelo Gargantini, and Marco Radavelli. 2019. Achieving change requirements of feature models by an evolutionary approach. fournal of Systems and Software 150 (2019), 64-76. https://doi.org/10.1016/j.jss.2019.01.045 